\title{
$T \bigcirc-110$
}

- March of Dimes award to Davor Solter Davor Solter, director of the Max-Planck-Institute in Freiburg, Germany and adjunct senior staff scientist at the Jackson Laboratory in Bar Harbor, Maine, will receive this year's March of Dimes prize for Developmental Biology. In honouring Solter for his pioneering work on genetic imprinting, the March of Dimes Foundation continues its quest to single out investigators who have made seminal discoveries in developmental biology that is relevant to human birth defects, and who have not previously received a major prize for their work. Among other discoveries, Solter developed the embryo fusion nucleartransfer technique which spawned much work on imprinting, but also re-invigorated attempts to clone mammalian embryos that ultimately led to the cloning of Dolly. "I am delighted that they picked Davor", said Robin Lovell-Badge of the National Institute for Medical Research in London, "he is a uniquely imaginative and charming scientist, a great lateral thinker and experimentalist. Someone who has no clones and who leaves an imprint on everyone he meets!". Solter is thinking about how to spend the prize money $(\$ 100,000)$ as "it's too much for a nice dinner and too little to retire immediately".

\section{The mosquito transformed}

With global warming forecast, signs that malarial parasites are developing immunity to traditional prophylactics, and a depressing record in the prophylaxis and treatment of vector-borne disease, the need for a means by which to control vector-mediated infection is pressing. Two recent studies (Proc. Natl Acad. Sci. USA 95, 3337-

3747 and $3748-3751,1998)$ provide a quantum leap forward,

in that they represent the first demonstrations of germline genetic change in Aedes aegypti, the mosquito that carries yellow-fever and dengue viruses. This may come as a surprise to those

with a focus on organisms (for example, Drosophila and the mouse) in which germline transmission of exogenously introduced genes as been taken for granted for some time. The mosquito has honed its elusive tactics in more ways than one. One of the barriers to effective transformation was the lack of suitable endogenous transposable elements - with respect to carrying exogenous genes. Groups led by Anthony James and Frank Collins (both of the University of California) have cleared this hurdle by tweaking the transposable elements, Mariner and Hermes, from other flies-the fruitfly and housefly, respectively. While there are of course, many more hurdles to be cleared before this technique can be safely applied as a means of vector control, these studies represent a breakthrough in a field that needs it.
OHFE fi fo fum...

... I smell the blood of some more haemochromatosis studies. These clarify the role of HFE in mediating the transport of iron (in the form of transferrin) through the cell membrane. The role of the protein was insinuated by the discovery that mutation of HFE is responsible for haemochromatosis, a disorder in which an excess of iron is carried in the blood and deposited in peripheral organs. Randi Schatzman (Progenitor, inc.) and colleagues have now demonstrated that HFE forms a complex with the transferrin receptor (TfR) - their data suggest that this interaction reduces transferrin uptake (Proc. Natl Acad. Sci. USA 95, 1472-1477, 1998). HFE is an MHC homologue and binds $\beta 2$ microglobulin; the most common HFE mutation abrogates HFE binding with $\beta 2$ microglobulin and does not appear at the cell surface. The effect of a second missense mutation, H63D, down-regulates HFE effect; Shatzman's data show that overexpression of wild-type HFE decreases the affinity of TfR for transferrin, but that of the H63D variant does not. In their report on the crystal structure of HFE, Pamela Bjorkman and colleagues (University of California) present data to suggest that $\mathrm{H} 63 \mathrm{D}$ effects a change in HFE tertiary structure. They also report that HFE and TfR bind tightly at the basic $\mathrm{pH}$ of the cell surface, but not at the acidic $\mathrm{pH}$ of intracellular vesicles, suggesting a role for HFE in trafficking iron and with implications for therapeutic intervention (Cell 93, 111-123, 1998). A meeting will be held at the National Institutes of Health on May 14-15 to review current art and mull on future investigations. Those interested should contact the conference manager, Tracy Moran, at Tracy@ccc76.com.
HFE (multicoloured ribbons) interacts with transferrin receptor (purple schematic) the cell surface, resulting in a lower cor affinity for ironFigure kindly provided figure kindly provided by Melanie Bennett and Pamela tute of Technology).

\section{IMAGE UNAVAILABLE FOR COPYRIGHT REASONS} Bjorkman (California Insti- 